\title{
Identifying Variable Number of Tandem Repeat Alleles in Phenylalanine Hydroxylase Gene in Patients with Phenylketonuria in Golestan Province, Iran
}

\author{
Golnaz Abedini ${ }^{1}(\mathbb{D})$, Zeinab Khazaei-Koohpar ${ }^{1 *(D)}$
}

1. Dept. of Cell and Molecular Biology, Faculty of Biological Sciences, Tonekabon Branch, Islamic Azad University, Tonekabon, Iran

\begin{tabular}{|c|c|}
\hline Article Info & ABSTRACT \\
\hline dof $10.30699 / j a m b s .28 .129 .198$ & \multirow{3}{*}{$\begin{array}{l}\text { Background \& Objective: Phenylketonuria (PKU) is a metabolic disorder that is } \\
\text { caused by mutations in the phenylalanine hydroxylase }(P A H) \text { gene. The multiplicity } \\
\text { of mutations in the } P A H \text { gene of PKU leads to the cases, in which the pathogenic } \\
\text { mutation cannot be detected. In these cases, the variable number of tandem repeat } \\
\text { (VNTR), which is the polymorphic marker associated with the } P A H \text { gene, is used } \\
\text { to identify PKU carriers. The present study was conducted to investigate the allele } \\
\text { frequency of this marker in PKU population of Golestan Province (north of Iran). }\end{array}$} \\
\hline $\begin{array}{l}\text { Received: 2020/05/30; } \\
\text { Accepted: 2020/07/15; } \\
\text { Published Online: } 31 \text { July 2020; }\end{array}$ & \\
\hline \multirow{2}{*}{$\begin{array}{l}\text { Use your device to scan and read the } \\
\text { article online }\end{array}$} & \\
\hline & Materials \& Methods: In this cross-sectional descriptive study, 26 non-relative \\
\hline \multirow{2}{*}{ 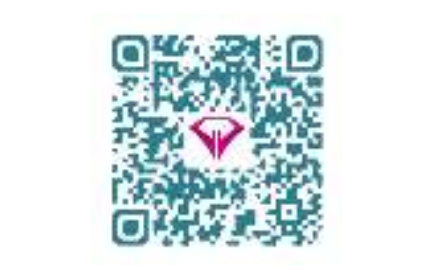 } & $\begin{array}{l}\text { PKU patients (1-23 years old, phenylalanine: } 4.5-250 \mathrm{mg} / \mathrm{dl}) \text { were identified from } \\
\text { different regions of Golestan Province within a one-year period (2016). Genomic } \\
\text { DNA was then extracted from leukocytes using the high pure PCR template } \\
\text { preparation kit (Roche) and the fragments containing the PAH VNTR were evaluated } \\
\text { using PCR-sequencing method. }\end{array}$ \\
\hline & $\begin{array}{l}\text { Results: PCR products of PAH VNTR alleles produced } 334,454,484,514 \text {, and } 604 \\
\text { bp fragments. They were corresponding to the presence of alleles with } 3,7,8,9 \text {, and }\end{array}$ \\
\hline \multirow{3}{*}{$\begin{array}{l}\text { Corresponding Information: } \\
\text { Zeinab Khazaei-Koohpar, } \\
\text { Dept. of Cell and Molecular Biology, } \\
\text { Faculty of Biological Sciences, } \\
\text { Tonekabon Branch, Islamic Azad } \\
\text { University, Tonekabon, Iran }\end{array}$} & was as follows: $28.85 \%, 28.85 \%, 17.3 \%, 19.23 \%$, and $5.77 \%$. \\
\hline & Conclusion: This study is the first report regarding the genetic structure of PKU \\
\hline & $\begin{array}{l}\text { population using PAH VNTR alleles in Golestan Province. Considering the } \\
\text { population diversity in Iran, it is necessary to investigate the frequency and } \\
\text { distribution of VNTR alleles in different parts of the country. }\end{array}$ \\
\hline E-Mail: $\underline{\text { dz.khazaei@gmail.com }}$ & Keywords: Phenylketonuria, Phenylalanine hydroxylase, VNTR, Iran \\
\hline & \\
\hline
\end{tabular}

\section{Introduction}

Phenylketonuria (PKU) is one of the most common inborn errors of amino acid metabolism with an autosomal recessive inheritance pattern $(1,2)$, which was first described by Folling in 1934 (3). PKU is the result of phenylalanine hydroxylase (PAH) deficiency that catalyzes the irreversible conversion of phenylalanine into tyrosine in the presence of the tetrahydrobiopterin (BH4) cofactor (4). PAH deficiency results in the abnormally elevated serum phenylalanine levels of more than $120 \mu \mathrm{mol} / \mathrm{L}$, which causes irreversible mental retardation in untreated patients (5). The $P A H$ gene is located on the long (q) arm of chromosome 12 in $\mathrm{q} 22-\mathrm{q} 24.1$ regions. This gene contains 13 exons and 12 introns (6). PKU has the prevalence of 1 per 10,000 births in Caucasians, but is considerably higher in the eastern Mediterranean region. In fact, the highest prevalence of PKU is reported in this region of the world: 1 per 4,000 in Turkey and 1 per 3627 in Iran. This high prevalence can be attributed primarily to the high rate of consanguineous marriage in the region (3). Another study also reported the highest prevalence in Turkey and the lowest prevalence in the UAE (7). Based on pre-treatment blood phenylalanine (Phe) levels, hyperphenylalaninemia and PKU are clinically divided into four groups: HPA (2-10 mg/dl), mild PKU (10$15 \mathrm{mg} / \mathrm{dl})$, moderate PKU (15-20mg/dl), and classic PKU (>20mg/dl) (8). PKU is often caused by mutations in the $P A H$ gene $(4,9)$. The $P A H$ locus database lists over $800 P A H$ mutations including missense and nonsense mutations, small and large deletions, as well as small insertions and splicing defects. This gene is located on chromosome 12 of humans. The cDNA sequence contains 13 exons and encodes 452 amino acids (4). The $P A H$ gene has various polymorphic markers such as intragenic short tandem repeats (STRs) located in intron 3, intragenic variable number of tandem repeats (VNTR) located in the $3^{\prime}$ untranslated regions ( $3^{\prime}$-UTR), and several restriction fragment length polymorphisms (RFLPs), which are 
highly associated with the $P A H$ region (1). Multiallelic VNTR polymorphism has an AT-rich repeat unit of $30 \mathrm{bp}$ and several alleles of this polymorphism have been identified and reported in the world (3). Polymorphic markers that are strongly linked to the $P A H$ gene facilitate the prenatal diagnosis and identification of carriers and are also important in detecting the haplotypes in the $P A H$ gene and tracking the geographical origin of the mutations (10). Many studies have been performed regarding the tandem repeat sequences of $P A H$ VNTR gene thus far. In these studies, polymorphism of this sequence, its association with a variety of mutations in the $P A H$ gene, ability of this sequence in identifying PKU carriers, and use of the VNTR sequence alongside repeat STR sequences in forensic identification have also been considered $(1,3,5)$. Overall, polymorphic systems have a high degree of heterogeneity and are highly informative for the prenatal diagnosis and carrier screenings in most of the populations (11). According to studies conducted in Iran, the high heterozygosity rate of the VNTR has provided a $66 \%$ information rate for the Iranian population (3). Given the high prevalence of PKU and consanguineous marriages in the Iranian population (12), the present study was conducted to identify $P A H$ VNTR alleles in PKU patients in Golestan Province, located in the north of Iran.

\section{Materials and Methods}

\section{Patients}

This study was approved by Ethics Committee of Golestan University of Medical Sciences and Health Services with the confirmation code: IR.GOUMS.REC.1394.204. The present study was a cross-sectional and descriptive work, in which 26 nonrelative PKU patients were identified from different parts of Golestan Province within a one-year period (2016). These patients were identified based on their records at Taleqani Hospital in Gorgan. Only one patient was included from each family and the patients with $\mathrm{BH} 4$ deficiency were excluded. The pre-treatment Phe levels were determined to be $4.5-250 \mathrm{mg} / \mathrm{dl}$ in 24 patients and their levels were not available in two patients $(7.7 \%)$. Then, the patients and their families were invited to participate in this study. After justifying the participants, consent forms and questionnaires were completed by the patients or their families (in case where the patient was a child or had mental retardation). First, 2-5 mL blood samples were collected from each patient and, to prevent blood coagulation, Falcons $15 \mathrm{~mL}$ containing $300 \mu \mathrm{L}$ of $0.5 \mathrm{M}$ EDTA was used as the anticoagulant.

\section{DNA Extraction}

High pure PCR template preparation kit (Roche, Germany) was used for DNA extraction according to the manufacturer's instructions. After DNA extraction and before PCR reaction, the obtained DNA was analyzed by a Nano Spectrophotometer (Thermo
Scientific NanoDrop, 2000C, USA) to evaluate the quantity and quality of nucleic acid.

\section{Polymerase Chain Reaction (PCR)}

The PCR was performed on a thermocycler (TC4000, TECHNE, UK). The primers used in this study were designed in Oligo7 software. The sequence of the forward and reverse primers employed in this reaction was as follows:

Forward primer $\left(5^{\prime} \rightarrow 3^{\prime}\right)$ :

\section{AAACTTAAGAATCCCATCTCTCAGAG}

Reverse primers $\left(5^{\prime} \rightarrow 3^{\prime}\right)$ :

\section{GATTTTAATGTTCTCACCCGCC}

The primers were synthesized by Bioneer Company (South Korea).

The length of the final PCR product was $484 \mathrm{bp}$ and the final volume of PCR mixture was $50 \mu \mathrm{L}$, which was prepared by Taq PCR Mix 2x kit (Biotechrabbit, Germany) and via adding genomic DNA, primer pairs (20 pmol), and sterile water to PCR mixture. The results of modifying PCR conditions for amplification of $P A H$ VNTR region were as follows: initial denaturation temperature of $95^{\circ} \mathrm{C}$ for 5 minutes and 33 reaction cycles each including: denaturation temperature of $95^{\circ} \mathrm{C}$ for 15 seconds, primers adhesion temperature of $60^{\circ} \mathrm{C}$ for 10 seconds, elongation temperature of $72^{\circ} \mathrm{C}$ for 40 seconds, and final elongation temperature of $72^{\circ} \mathrm{C}$ for 5 minutes.

\section{Electrophoresis of PCR Products}

To ensure the amplification of the desired fragment and its quality and not amplifying the non-specific products, $3 \mu \mathrm{L}$ of the reaction products (patient samples) and $100 \mathrm{bp}$ marker (100bp DNA Ladder, BR0800201, Biotechrabbit, Germany) were loaded and electrophoresed on the $2 \%$ agarose gel.

\section{Sequencing of PCR Products}

After viewing a clear DNA band on the gel, the remainder of the PCR product was sent to Bioneer Company. (South Korea) for sequencing. Sequencing was performed by the ABI 3730 DNA Sequencer using the chain termination method.

\section{Applications}

Chromas and CLC main workbench v3.5 applications were utilized to analyze the results of the samples sequencing data.

\section{Results}

\section{Patient Phenotype}

In this study, 26 HPA patients referring to Taleqani Hospital in Gorgan were examined by a pediatric endocrinologist. Patients were divided into five groups according to pretreatment Phe levels (Table 1). Patients 
(26 patients) were in the age range of 1 to 23 years old and their ethnic composition included Fars (20 patients, $76.9 \%$ ), Turkmen (5 patients, 19.2\%), and Lor (1 patient, 3.8\%). These patients were identified based on their records at Taleqani Hospital in Gorgan, Iran.

Table 1. Frequency of the disease in the study population based on pretreatment phenylalanine concentration

\begin{tabular}{cc} 
\%Patients & Metabolic phenotype \\
$57.7 \%$ & Classic PKU \\
$11.5 \%$ & PKUModerate \\
$7.7 \%$ & Mild PKU \\
$15.4 \%$ & HPA \\
$* 7.7 \%$ & Unknown \\
\hline
\end{tabular}

* Patients whose records were incomplete.

\section{Quantitative and Qualitative Results of Extracted DNA}

Nano Spectrophotometer showed the quantity and quality of DNA extracted from the blood samples of patients to be in the range of $100-1000 \mathrm{ng} / \mu \mathrm{L}$ and absorption ratio of $260 / 280$ in the range of $1.8-2$, respectively. The DNA extracted with the mentioned quantity and quality was appropriate for PCR.

\section{Results of Gel Electrophoresis of PCR Products:}

The electrophoresis image of PCR products on $2 \%$ agarose gel is shown in Figures $1(\mathrm{~A}, \mathrm{~B}, \mathrm{C})$. The PCR products of the $P A H$ VNTR alleles produced $334,454,484,514$, and 604 bp fragments. They were corresponding to the presence of alleles with $3,7,8,9$, and 12 copies of the repeat units, respectively. In our study, of 52 analyzed alleles, alleles corresponding to $3,7,8,9$, and 12 repeats had frequencies of 15 (28.85\%), 15 (28.85\%), 9 (17.3\%), 10 (19.23\%), and 3 $(5.77 \%)$, respectively. Table 2 lists the number and frequency of alleles related to the studied patients. In addition, the frequency of heterozygous and homozygous states in the studied population included VNTR3 / VNTR7 (11.5\%), VNTR3 / VNTR8 (3.84\%), VNTR3 / VNTR12 (3.84\%), VNTR3 / VNTR3 (19.23\%), VNTR7 / VNTR7 (23\%), VNTR8 / VNTR8 (15.38\%), VNTR9 / VNTR9 (19.23\%), and VNTR12 / VNTR12 (3.84\%) (Figure $1(\mathrm{~A}, \mathrm{~B}, \mathrm{C})$ ).

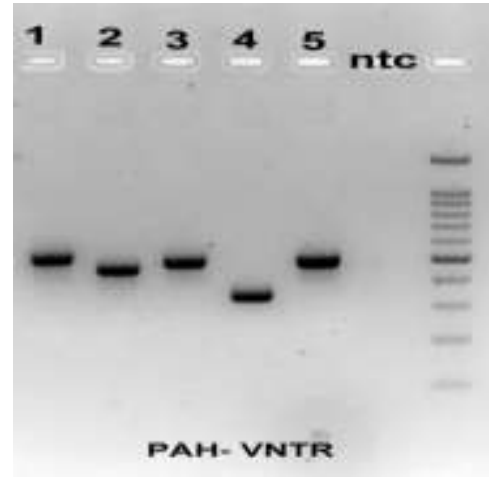

A

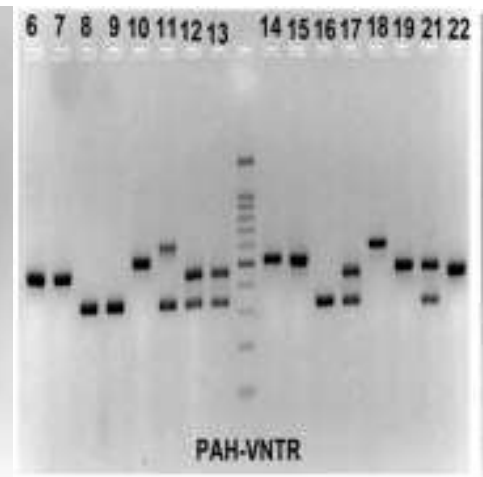

B

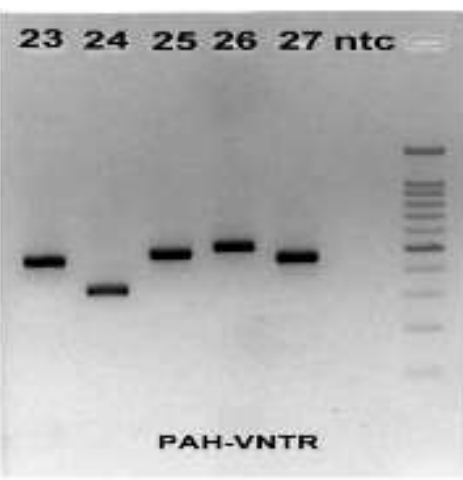

$\mathrm{C}$

Figure 1. Images of PAH VNTR alleles on $2 \%$ agarose gel for 26 samples, A:well1:(514bp/514bp),well2:(454bp/454bp),well3:(484bp/484bp),well4:(334bp/ 334bp),well5:(484bp/484bp),NTC: negative control ,well -:100 bp molecular marker. B:well 6: (454bp/454bp), well 7: (454bp/454bp), well 8: (334bp/334bp), well 9: (334bp/ 334bp),well10: (514bp/ 514bp),well 11: (604bp/334bp),well 12: (454bp/334bp),well 13: (454bp/334bp),well -:100 bp molecular marker,well14:(514bp/514bp),well15:(514bp/514bp),well16:(334bp/334bp),well17:(454bp/334bp),well18:(604bp/604bp),well1 9:(484bp/484bp),well21:(484bp/334bp),well22:(454bp/454bp).C:well23:(454bp/454bp),well24:(334bp/334bp),well25:(484bp/4 84bp),well26:(514bp/514bp),well27:(454bp/454bp),NTC:negative control, well -:100 bp molecular marker.

\section{Sequencing Results}

After PCR reaction, the samples were sequenced to confirm the number of repeats in the VNTR alleles.
The base sequence of the PAH VNTR alleles was determined in Chromas software and CLC main workbench v3.5; this way, the number of repeats in the VNTR alleles was specified (Table 2).

Table 2. Number and frequency of PAH VNTR alleles observed in PKU population of Golestan province, northern Iran 


\begin{tabular}{|ccccccc} 
Percent & Total & Lor & $\begin{array}{c}\text { Number of chromosomes } \\
\text { In the situations studied } \\
\text { Turkmen }\end{array}$ & Fars & VNTR alleles & $\begin{array}{c}\text { PCR product } \\
\text { length(bp) }\end{array}$ \\
\hline $28.85 \%$ & 15 & 1 & 2 & 12 & 3 & 334 \\
\hline $28.85 \%$ & 15 & - & 4 & 11 & 7 & 454 \\
\hline $17.3 \%$ & 9 & - & - & 9 & 8 & 484 \\
\hline $19.23 \%$ & 10 & - & 4 & 6 & 9 & 514 \\
\hline $5.77 \%$ & 3 & 1 & - & 2 & 12 & 604 \\
\hline
\end{tabular}

\section{Discussion}

In this study, the VNTR alleles were investigated in the $P A H$ gene in 26 patients with phenylketonuria in Golestan Province for the first time. PCR sequencing was used in this study. The results of analyzing 52 alleles led to identifying 5 VNTR alleles that were related to $3,7,8,9$, and 12 copies of the repeat units with frequencies of $15(28.85 \%), 15(28.85 \%), 9$ $(17.3 \%), 10(19.23 \%)$, and $3(5.77 \%)$, respectively. Furthermore, VNTR with 3 and 7 repeat units had the highest frequency in our study. It is noteworthy that VNTR with 3 repeats was observed in all the three ethnic groups of Fars, Turkmen, and Lor, and the highest frequency belonged to Fars ethnic group.

There have been several studies about VNTR alleles in the $P A H$ gene in Iranian families with PKU; in the study by Hosseini-Mazinani et al. in 2008, four VNTR alleles were identified on PKU chromosomes, in which the distribution of alleles on PKU chromosomes was VNTR3 (7.1\%), VNTR7 (31.3\%), VNTR8 (48.3\%), and VNTR9 (13.3\%). However, VNTR12 allele $(0 \%)$ was not found in their study (3). In our investigation, in addition to the above-mentioned four alleles, the VNTR12 allele was observed with the frequency of $5.77 \%$. In another study conducted in 2011 by Parivar et al. on the population of Yazd Province, four VNTR alleles with 3, 7, 8, and 12 repeats were found in the PKU chromosomes with frequencies of $5.5 \%, 11 \%$, $78 \%$, and $5.5 \%$, respectively, and the highest frequency was related to VNTR allele with 8 repeats. Nevertheless, in comparison with PKU population of Golestan Province (the present study), allele 9 was not found in PKU chromosomes of Yazd Province (10). In addition, Bagheri et al. (2015) reported five alleles of VNTR3 (15.1\%), VNTR7 (3.49\%), VNTR8 (74.4\%), VNTR $9(5.81 \%)$, and VNTR $11(1.16 \%)$ among the Iranian Azeri Turkish patients with phenylketonuria (PKU), while the highest frequency $(74.4 \%)$ belonged to VNTR8 allele (13).

On the other hand, in a study by Razipour et al. (2017) among 81 PKU patients from different parts of Iran, different mutations and mini-haplotypes were identified. Five VNTR alleles with 3, 7, 8, 9, and 12 repeats were reported in their study (1), which was consistent with our findings in Golestan Province. Subsequently, 18 non-relative PKU patients from three provinces of Kermanshah, Hamadan, and Lorestan were investigated in terms of $P A H$ gene mutations and their association with VNTR alleles by Alibakhshi et al. (2018). They detected 11 specific mutations and 4 VNTR alleles with 3, 7, 8, and 9 repeats (4). However, in comparison with Golestan Province, their study did not show VNTR allele with 12 repeats. In addition, another investigation was conducted in 2018 on 25 non-relative PKU patients in Gilan Province, northern Iran, in which 3 VNTR alleles were identified with 3, 7 , and 8 repeats while the highest frequency $(60 \%)$ belonged to VNTR allele with 8 repeats (data were not published). However, Golestan Province (in the present study), which is another province in northern Iran, had more diversity of VNTR alleles and VNTR alleles with 3 and 7 repeats had the highest frequency.

On the other hand, the VNTR alleles identified in our study (in PKU patients of Golestan Province) corresponded to alleles previously described among the European white families (3). VNTR12, which accounts for $4.3 \%$ of PKU alleles in European white people, was about $5.77 \%$ in our study, whereas the diversity in this polymorphic locus was higher in the samples compared to Chinese PKU families, in which only alleles of VNTR with 3 and 8 repeats were reported (14). The frequency of VNTR3 and VNTR7 on PKU chromosomes in Golestan Province was significantly different from reports of European population; VNTR3 showed lower frequency and VNTR7 showed higher frequency in Golestan Province than the European population. This is partly attributable to the different mutation spectra in these populations. The R408W mutation is the most common mutation that causes PKU in European populations and is associated with VNTR3 allele, especially in the eastern parts of this continent (15). This mutation is very rare in the Iranian population. On the other hand, the Mediterranean IVS10nt546 mutation has been associated with VNTR7, which is the most common mutation in Iran $(16,17)$. This mutation was also highly prevalent in Golestan Province $(18,19)$. Due to the lack of funding for investigating another polymorphic marker (STR) associated with the $P A H$ gene in PKU patients, in the present work, only the VNTR marker was considered in these patients. 


\section{Conclusion}

Due to population variation in Iran, the type of VNTR alleles in the $P A H$ gene may vary in different regions. Therefore, it is necessary to study the frequency and distribution of VNTR marker alleles in different regions of the country. In general, this study was a preliminary work for future practical use of this sequence (VNTR) for various purposes such as identification of heterozygous individuals regarding phenylketonuria in the community and analysis of gene flow in the population.

\section{Acknowledgments}

This article is the result of a Master's thesis in Cellular and Molecular Sciences from Islamic Azad University- Tonekabon Branch. Hereby, we would like to thank Dr. Hossein Zarei Aghmashhadi and the staff of Taleqani Hospital in Gorgan who contributed to the research.

\section{Conflict of Interest}

Authors declared no conflict of interest.

\section{References}

1. Razipour M, Alavinejad E, Sajedi S Z, et al. Genetic study of the PAH locus in the Iranian population: familial gene mutations and minihaplotypes.Metabolic Brain Dis.2017, 32(5): 1685-91 [DOI:10.1007/s11011-017-0048-7]

2. Shaykholeslam Esfahani M, Vallian S. A comprehensive study of phenylalanine hydroxylase gene mutations in the Iranian phenylketonuria patients. Eur J Med Genet. 2019;62(9):1-6 [DOI:10.1016/j.ejmg.2018.10.011]

3. Hosseini-Mazinani SM, Koochmeshgi J, Khazaee-Koohpar Z, Hossein-Pur-Nobari N, Seifati SM.. Carrier detection of phenylketonuria in Iranian families by variable number tandemrepeat polymorphism analysis. East Mediterr Health J,2008; 14: 1445-51

4. Alibakhshi R., Moradi K., Biglari M., Shafieenia S. Spectrum of phenylalanine hydroxylase gene mutations in Hamadan and Lorestan provinces of Iran and their associations with variable number of tandem repeat alleles. Iran J Med Sci.2018;43(3):318-23.

5. Bagheri M, Abdi Rad I, Hosseini Jazani N,Zarrin R,Ghazavi A. Association between PAH mutations and VNTR alleles in the west azerbaijani PKU patients . MAEDICA, J Clin Med.2014; 9(3): 242-47.
6. Pourvatan N, Khazaei -Koohpar Z.Investigation of exon 4 mutations in phenylalanine hydroxylase gene in phenylketonuria patients from Guilan province using PCR-Sequencing. Feyz J Kashan Uni Med Sci; 2019. 22(6):595-601.

7. Jafarzadeh-Esfehani R, Vojdani S, Hashemian S, Mirinezhad M, Pourafshar M, Forouzanfar N. Genetic variants of the phenylalanine hydroxylase gene in patients with phenylketonuria in the northeast of Iran. J Pediatr Endocrinol Metab. 2020; aop,1-5.

8. Gundorova P, Zinchenko RAP, Makarov Akh, Polyakov AV,The spectrum of mutations in the $\mathrm{PAH}$ gene in patients with hyperphenylalaninemia from the karachaycherkess Republic Russ J Genet ;2017. 53(7):81319 [DOI:10.1134/S1022795417070043]

9. Madadi N, Khazaei Koohpar Z, Ranji N.Molecular analysis of exon 6 mutations of phenylalanine hydroxylase gene in phenylketonuria patients from Guilan province .Razi J Med Sci.2019;26(1):32-39.

10. Parivar K, Seifati M, Koochmeshgi J, Hashemi M. Studying the level of informativity of VNTR marker on PAH gene for carrier detection of the patients with phenylketonuria in Yazd province, Iran.J Tehran Azad Med Univ .2011; 21(3): 196200.

11. Scriver CR,Eisensmith RC,Woo SLC,Kaufman's:The hyperphenylalaninemia of man and mouse. Ann Rev Genet.1994,28:141-65. [DOI:10.1146/annurev.ge.28.120194.001041]

12. Koochmeshgi J, Bagheri A, Hosseini-Mazinani SM. Incidence of phenylketonuria in Iran estimated from consanguineous marriages. Inherit Metab Dis .2002; 25(1): 80-1 [DOI:10.1023/A:1015154321142]

13. Bagheri M,Abdi Rad I, Hosseini Jazani N,Zarrin R,Ghazavi A. Frequency of the VNTRpolymorphisms at the PAH gene in the Iranian Azeri Turkish patients with phenylketonuria. MAEDICA . 2015; 10(4): 310-14.

14. Eisensmith RC, Goltsov AA, Woo SLC.A simple, rapid, and highly informative PCR-based procedure for prenatal diagnosis and carrier screening of phenylketonuria. Prenatal Diag, 1994,14(12):1113-8 [DOI:10.1002/pd.1970141204]

15. Tighe O, Dunican D, O'Neill C, et al. Genetic diversity within the R408W phenylketonuria mutation lineages in Europe. Human Mutation; $2003.21(4): 387-93$ [DOI:10.1002/humu.10195] 
16. Zare-Karizi S, Hossini-Mazinani SM, Khazaei koohpar Z, et al. Mutation spectrum of phenylketonuria in Iranian population. Mol Genet Metab.2011;102(1): 29-32

[DOI:10.1016/j.ymgme.2010.09.001]

17. Shirzad T, Saeidian AH, Bagherian H, et al. Molecular genetics of a cohort of 635 cases of phenylketonuria in a consanguineous population . J Inherit Metab Dis. 2018;1-9 [DOI:10.1007/s10545-018-0228-6]
18. Haerian Ardakani $H$, Khazaei Koohpar Z,Mohammadian S. Mutations analysis of exon 10-11 of phenylalanine Hydroxylase gene in phenylketonuria patients from Golestan province. Razi J Med Sci; 2018. 25(172):38-46.

19. Zamanfar D,Jalali H,Mahdavi M R,Maadanisani $\mathrm{M}$, Zaeri H,Asadpoor E.Investigation of five common mutations on phenylalanine hydroxylase gene of phenylketonuria patients from two provinces in north of Iran. Int $\mathbf{J}$ Prev Med .2017;8:89:1-4

\section{How to Cite This Article:}

Abedini G, Khazaei Koohpar Z. Identifying Variable Number of Tandem Repeat Alleles in Phenylalanine Hydroxylase Gene in Patients with Phenylketonuria in Golestan Province, Iran. J Adv Med Biomed Res. 2020; 28 (129) :198-203

\section{Download citation:}

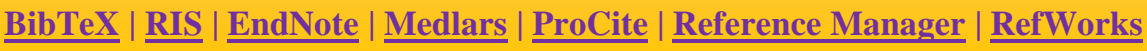

\section{Send citation to:}

90. Mendeley Zotero PefWorka RefWorks 